\title{
FATORES DE RISCO PARA DOENÇAS CRÔNICAS NÃO TRANSMISSÍVEIS, ENTRE ALUNOS DE ENFERMAGEM DE RIBEIRÃO PRETO-BRASIL
}

RISK FACTORS FOR NON-COMMUNICABLE DISEASES IN NURSING STUDENTS FROM RIBEIRÃO PRETO-BRAZIL

\author{
Suzana Alves de Moraes ${ }^{1}$; Luciana Meira² \& Isabel Cristina Martins de Freitas ${ }^{3}$
}

1Docente do Departamento de Enfermagem Materno-Infantil e Saúde Pública da Escola de Enfermagem de Ribeirão Preto-USP (EERP-USP). ${ }^{2}$ Bolsista de Aperfeiçoamento do Programa CNPq da EERP-USP. ${ }^{3}$ Membro Efetivo do Núcleo de Epidemiologia - NEPI da EERP-USP.

CorrespondÊncia: Suzana Alves de Moraes. Av.Santa Luzia No.440. Apto. 81. Sumaré - CEP 14025-090 Ribeirão Preto-SP

MORAES SA; MEIRA L \& FREITAS ICM. Fatores de risco para doenças crônicas não transmissíveis; entre alunos de enfermagem de Ribeirão Preto-Brasil. Medicina, Ribeirão Preto, 33: 312-321, jul./set. 2000.

RESUMO: Objetivo: o estudo teve por objetivo analisar a prevalência de alguns fatores de risco para as doenças crônicas não transmissíveis, entre estudantes de Enfermagem da USP de Ribeirão Preto, investigando-se o efeito independente de alguns desses fatores sobre os níveis de pressão arterial sistólica e diastólica.

Material e Métodos: a população estudada foi constituída por 305 alunos, matriculados em 1995, e a coleta de dados ocorreu entre agosto e dezembro do mesmo ano, mediante uma entrevista padronizada, realizada por uma equipe treinada. Além de condições sociodemográficas, foi investigada a prevalência de antecedentes familiares para doenças crônicas não transmissíveis, dos níveis de pressão arterial, do hábito de fumar, do índice de massa corporal e do uso de contraceptivos orais. A prevalência dos fatores de risco foi estimada por ponto e por intervalos que foram construídos com $95 \%$ de confiança. Para o estudo de associação, foi utilizada a técnica de regressão linear múltipla, obtendo-se os estimadores $(\beta)$ dos coeficientes de regressão, com testes de hipóteses e o nível de significância adotado foi a $\leq 0,05$.

Resultados: com relação à presença de antecedentes familiares, predominaram aqueles indivíduos com dois ou mais antecedentes familiares com doenças crônicas não transmissíveis, $65,6 \%$ (IC: $59,9 \% 0150-70,8 \%$ ). A prevalência do hábito de fumar foi $15,4 \%$ (IC:11,6\%-20,1\%) e a prevalência do uso de contraceptivos orais, entre as mulheres, foi $48,6 \%$ (IC: $42,7 \%-54,6 \%$ ). $O$ índice de massa corporal (IMC), ajustado para idade, e hábito de fumar (conjunto dos indivíduos) ou para idade, uso de contraceptivos orais ou sua duração (população feminina), destacou-se como um forte preditor da pressão sistólica e diastólica $(p<0,05)$. O IMC, a duração do hábito de fumar e o número de cigarros consumidos/dia, quando estratificados segundo as categorias de antecedentes familiares, apresentaram-se como bons preditores da pressão sistólica para aqueles indivíduos com história de dois ou mais antecedentes familiares $(p<0,05)$. Com relação aos níveis de pressão diastólica, seus melhores preditores foram o IMC entre aqueles com dois ou mais antecedentes familiares ou entre aqueles com apenas antecedentes de hipertensão arterial $(p<0,05)$. O número de cigarros consumidos/dia foi bom preditor dos níveis de pressão diastólica entre os indivíduos com antecedentes familiares apenas para cardiopatias $(p<0,05)$.

Discussão: discutem-se as implicações de fatores de ordem genética ou ambiental como preditores de doenças crônicas na população em estudo, recomendando-se estratégias efetivas de combate ao tabagismo, de controle da obesidade e de uso de contraceptivos orais nessa falange universitária.

UNITERMOS: Prevalência. Fatores de Risco. Doença Crônica . 


\section{1- INTRODUÇÃO}

Estudos multicêntricos, realizados recentemente, no Brasil e na América Latina, sobre fatores de risco para as doenças crônicas não transmissíveis têm apontado como causa para a alta prevalência para a hipertensão arterial sistêmica, sedentarismo, hábito de fumar, hipercolesterolemia e diabetes mellitus ${ }^{(1,2,3)}$.

Sabendo-se que as doenças crônicas não transmissíveis se caracterizam por sua longa latência, que, para uma mesma doença, pode haver participação de diversos fatores de risco e, que um mesmo fator de risco pode estar associado com múltiplas condições, vários pesquisadores têm se preocupado em investigar a prevalência desses fatores, em populações jovens, com o propósito de que medidas de prevenção e promoção à saúde possam ser efetivamente implementada ${ }^{(4,5,6)}$.

Beral et al., $1999^{(7)}$, detectaram um efeito independente do uso de contraceptivos orais sobre a mortalidade por câncer cervical e a doença cerebrovascular. Timmer et al.,1998 ${ }^{(8)}$, em uma coorte prospectiva encontraram associação entre o hábito de fumar e recidiva de doença de Crohn, sendo relatado, no mesmo estudo, um efeito independente do uso de contraceptivos orais sobre a recidiva da doença. Sanchez-Guerrero et al., $1997^{(9)}$, em outro estudo de coorte, encontraram associação entre o uso de contraceptivos orais e o desenvolvimento de Lúpus Eritematoso Sistêmico.

Moraes \& Souza, $1996^{(10)}$,em estudo de casocontrole, realizado no município de São Paulo, em 1993, detectaram um efeito independente do fumo, da hipertensão arterial e da hipercolesterolemia sobre a doença isquêmica do coração.

Estudos recentes sobre a prevalência do hábito de fumar em estudantes da área de saúde têm detectado a alta prevalência deste hábito entre os alunos e, principalmente, entre aqueles matriculados em cursos de enfermagem ${ }^{(11,12)}$.

Sob a ótica da epidemiologia analítica (estudos etiológicos), tem sido cada vez mais incentivada a procura dos mecanismos de atuação conjunta dos fatores de risco sobre um determinado evento resposta, ressaltando-se, ao lado de sua plausibilidade biológica na determinação de um dado desfecho, a aplicação de algumas técnicas estatísticas que permitem identificar fatores de confusão e/ou modificadores de efeito, sendo pressuposto, para as duas últimas condições, que tais fatores, além de associados com o eventoresposta, também estejam associados entre $\mathrm{si}^{(13)}$.

Levando-se, pois, em consideração a importância desses fatores na gênese das doenças crônicas não transmissíveis, o presente estudo teve por objetivo estudar a prevalência de alguns fatores de risco para as doenças crônicas, entre estudantes do Curso de Enfermagem da USP de Ribeirão Preto, investigando-se o efeito independente de alguns desses fatores de risco sobre os níveis de pressão arterial sistólica e diastólica.

\section{2- MATERIAL E MÉTODOS}

\section{- Delineamento do Estudo}

O estudo foi planejado sob a forma de um desenho do tipo transversal, um dos delineamentos clássicos em pesquisa observacional ${ }^{(13)}$.

\section{- População do Estudo}

A população estudada foi constituída por 305 alunos, matriculados no $2^{\circ}$ semestre de 1995 , nas quatro séries escolares do curso de Graduação da Escola de Enfermagem de Ribeirão Preto. De um universo de 337 alunos, apenas 32 se recusaram a participar do estudo, constituindo uma perda equivalente a $9,5 \%$.

\section{- Local e período do estudo}

A coleta de dados foi efetuada entre agosto e dezembro de 1995, e o estudo foi desenvolvido na Escola de Enfermagem de Ribeirão Preto da Universidade de São Paulo.

\section{- Variáveis estudadas e forma de categorização}

\section{1) Sexo e Idade}

Foram considerados ambos os sexos. A idade foi coletada em anos completos e tratada como variável contínua.

\section{2) História Familiar}

Foram coletadas informações sobre antecedentes familiares de diabetes mellitus, cardiopatias, hipercolesterolemia e hipertensão arterial, investigadas com base na presença da condição, previamente diagnosticada, entre os parentes consangüíneos (pais, tios e avós) de cada entrevistado. Essa variável foi classificada em sete categorias: a) sem antecedentes familiares; b) presença de antecedentes familiares para apenas uma das quatro condições; c) presença de dois ou mais antecedentes familiares para condições diferentes; d) ignorado para um ou mais antecedentes familiares, considerando as quatro condições investigadas.

\section{3) Pressão Arterial}

A pressão arterial foi aferida em cada um dos entrevistados, utilizando-se esfigmomanômetros 
anaeróides, previamente calibrados e utilizados exclusivamente para a pesquisa. Os indivíduos foram mantidos em repouso e as aferições realizadas no membro superior esquerdo. Foram obtidas três medidas consecutivas de pressão arterial, tomadas pelo mesmo entrevistador, com intervalo de um minuto entre as aferições, considerando-se, respectivamente, o primeiro e último ruído de Korotkoff para a definição da pressão sistólica e diastólica. As medidas foram tomadas em condições padronizadas, respeitando-se o intervalo mínimo de cinco minutos entre o início da entrevista e as aferições. Foi considerada a respectiva média das três medidas para a pressão sistólica e diastólica em cada indivíduo. Para os fumantes que fizeram uso do cigarro antes das entrevistas, estabeleceu-se um intervalo mínimo de 60 minutos antes das aferições.

Essa variável foi classificada em quatro categorias, segundo os respectivos quartis da amostra para a pressão sistólica e diastólica. Posteriormente, a variável foi tratada como contínua.

\section{4) Hábito e fumar}

O hábito de fumar foi investigado, levando-se em consideração a história atual ou pregressa do hábito de fumar cigarros. Os indivíduos foram classificados em "não fumantes"; "ex-fumantes"; e "fumantes", sendo que os fumantes foram subagrupados em fumantes habituais e ocasionais. Para os fumantes e ex-fumantes, foram também considerados a duração efetiva do hábito, em anos, e o número de cigarros consumidos/dia, sendo ambas as variáveis tratadas como contínuas.

5) Índice de massa corporal

O índice de massa corporal (IMC) foi calculado através da razão: peso/altura ${ }^{2}$. As medidas antropométricas foram aferidas sob condições padronizadas em balanças e estadiômetros previamente calibrados. Utilizando os critérios recomendados por Bray ${ }^{(14)}$, os indivíduos foram classificados em "normais" (IMC: <25); "sobrepeso" (IMC:25-29,9) e "obesos" (IMC: $\geq 30$ ). Posteriormente, o índice de massa corporal foi tratado como variável contínua.

\section{6) Uso de contraceptivos orais}

O uso de contraceptivos orais em indivíduos do sexo feminino foi investigado com base na história atual ou pregressa do uso, sendo as mulheres classificadas em "usuárias" e "não usuárias". Entre as usuárias, foi também considerado o tempo de uso em anos, o que foi tratado como variável contínua.

\section{- Instrumento de coleta de informações}

A coleta de informações foi procedida através de entrevistas em que um questionário pré-codificado foi aplicado e preenchido por uma equipe de entrevistadores previamente treinada. Para as medidas antropométricas e medida da pressão arterial, foram utilizados equipamentos da própria Instituição. As entrevistas foram realizadas em um laboratório da Instituição, em horários definidos para esse fim.

\section{- Processamento dos dados}

Os códigos originais, atribuídos às variáveis e constantes, no questionário, foram transportados para uma planilha de codificação previamente desenhada segundo a estrutura do banco de dados formatado no software FOXPRO ${ }^{(15)}$ vs. 2.06 para microcomputador. A seguir, os dados constantes das planilhas foram digitados no computador.

\section{- Análise estatística}

Para a obtenção da prevalência e respectivos intervalos de confiança (95\%) dos fatores de risco estudados, utilizou-se o módulo "ANALYSIS" do software EPIINFO $^{(16)}$ vs. $6.04 \mathrm{c}$ para microcomputador. Para o estudo de associação, utilizou-se a regressão linear múltipla $^{(17)}$ sendo os dados analisados no programa SPSS for Windows ${ }^{(18)}$. O nível de significância estabelecido foi $\mathrm{a} \leq 0,05$.

\section{- Considerações éticas do estudo}

Todas as informações procedentes das entrevistas foram resguardadas. A participação no estudo foi voluntária, não havendo nenhuma sanção aos não participantes.

\section{3- RESULTADOS}

A população do estudo foi constituída, essencialmente, por mulheres $(97,3 \%)$. A média de idade foi 22,1 e o desvio padrão foi 4,1 .

Os valores brutos e respectivos intervalos de confiança (95\%) para a prevalência dos fatores de risco em estudo encontram-se nas Tabelas de I a V.

Na Tabela I, encontra-se a prevalência de antecedentes familiares de doenças crônicas não transmissíveis. Destaca-se a elevada prevalência de indivíduos com dois ou mais antecedentes familiares $(65,6 \%)$.Entre aqueles que relataram a presença de antecedentes familiares para apenas uma condição, destacou-se a presença de antecedentes de hipertensão arterial $(9,2 \%)$. 


\begin{tabular}{|lrcc|}
\hline \multicolumn{4}{|c|}{ Tabela I - Prevalência de antecedentes familiares e interval os de confiança (IC-95\%) } \\
\hline \multicolumn{1}{|c|}{ Antecedentes familiares } & $N$ & \multicolumn{1}{c|}{$(\%)$} & $1 \mathrm{C}-95 \%$ \\
\hline Sem antecedentes familiares & 26 & $(8.5 \%)$ & $5.7 \%-12.4 \%$ \\
Apenas de diabetes meilitus & 12 & $(3.9 \%)$ & $2.1 \%-7.0 \%$ \\
Apenas de Hipercolesterolemia & 11 & $(3.6 \%)$ & $1.9 \%-6.5 \%$ \\
Apenas de Cardionatias & 20 & $(6.6 \%)$ & $4.2 \%-10.1 \%$ \\
Apenas de Hibertensẫo Arterial & 28 & $(9.2 \%)$ & $6.3 \%-13.1 \%$ \\
Com dois ou mais antecedentes familiares & 200 & $(65.6 \%)$ & $59.9 \%-70.8 \%$ \\
Ignorado pi um ou mais antecedentes familiares & 8 & $(2.6 \%)$ & $1.2 \%-5.3 \%$ \\
Total & 305 & $(100 \%)$ & - \\
\hline
\end{tabular}

Nas Tabelas II e III, encontram-se as distribuições dos indivíduos nos respectivos quartis de pressão arterial sistólica e diastólica. Embora não tenham sido evidenciados indivíduos hipertensos, as respectivas distribuições de pressão sistólica e diastólica seguem um gradiente no grupo.

\begin{tabular}{|c|c|c|c|}
\hline PAS Quartis & N & (\%) & $1 \mathrm{C}-95 \%$ \\
\hline 69- $91 \mathrm{mmHa}$ & 82 & $(26.9 \%)$ & $22.1 \%-32.3 \%$ \\
\hline 92- $97 \mathrm{mmHa}$ & 75 & $(24.6 \%)$ & $19.9 \%-29.9 \%$ \\
\hline $98-103 \mathrm{mmHa}$ & 74 & $(24.3 \%)$ & $19.6 \%-29.5 \%$ \\
\hline $104-131 \mathrm{mmHg}$ & 74 & $(24.3 \%)$ & $19.6 \%-29.5 \%$ \\
\hline Total & 305 & $(100 \%)$ & - \\
\hline
\end{tabular}

\begin{tabular}{|c|c|c|c|c|}
\hline PAD Quartis & $N$ & $(\%)$ & \multicolumn{2}{|c|}{ IC- $-95 \%$} \\
\hline $30-53 \mathrm{mmHg}$ & 78 & $(25,6 \%)$ & $29,9 \%$ & $-30,9 \%$ \\
\hline $54-59 \mathrm{mmHg}$ & 101 & $(33,1 \%)$ & $27,9 \%$ & $-38,7 \%$ \\
\hline $60-63 \mathrm{mmHg}$ & 54 & $(17,7 \%)$ & $13,7 \%$ & $-22,6 \%$ \\
\hline $64-87 \mathrm{mmHg}$ & 72 & $(23,6 \%)$ & $19,0 \%$ & $-28,9 \%$ \\
\hline Total & 305 & $(100 \%)$ & & - \\
\hline
\end{tabular}

Nas Tabelas IV e V, encontram-se as prevalências do hábito de fumar e do índice de massa corporal. Observa-se, para a categoria "fumantes habituais", uma freqüência de 15,4\% que, se somados aos "fumantes ocasionais", resultaria numa prevalência de $22 \%$. Para o indicador de obesidade, os indivíduos foram agrupados em três categorias: 1) normais, 2) sobrepeso e 3 ) obesos. Destaca-se que a maioria dos indivíduos (83\%) foi classificada como normal, seguidos daqueles com sobrepeso $(14,4 \%)$ sendo a obesidade detectada em apenas $2 \%$ da população analisada.

A prevalência de uso de contraceptivos orais, entre as mulheres, encontra-se na Tabela V. Observase que $48,6 \%$ da população feminina eram usuárias de contraceptivos.

Nos Quadros I e II, encontram-se os resultados das análises de regressão linear múltipla. $\mathrm{O}$ conjunto de variáveis: Idade, Hábito de Fumar e IMC, para ambos os sexos, esteve associado aos níveis de pressão sistólica $(\mathrm{p}<0,05)$. Com exceção do hábito de fu$\operatorname{mar}(\mathrm{p}>0,05)$, as demais variáveis se mantiveram associadas com os níveis de pressão diastólica. Quando as três variáveis foram consideradas para a população feminina, observou-se que apenas o IMC manteve-se associado com os níveis de pressão sistólica e diastólica $(\mathrm{p}<0,05)$. Quando consideradas as variáveis específicas para essa população, como o Uso de Contraceptivos Orais e sua Duração, mantendo-se a Idade e o IMC nos modelos, apenas o IMC apresentou associação com o níveis de pressão sistólica e diastólica $(\mathrm{p}<0,05)$. 


\begin{tabular}{|c|c|c|c|}
\hline & $N$ & $(\%)$ & $1 \mathrm{C}-95 \%$ \\
\hline \multicolumn{4}{|l|}{ Hábito de Fumar } \\
\hline Nẫo Fumantes & 214 & $(70,2 \%)$ & $64,6 \%-75,2 \%$ \\
\hline Ex-fumantes & 24 & $(7,9 \%)$ & $5,2 \%-11,6 \%$ \\
\hline Fumantes ocasionais & 20 & $(6,6 \%)$ & $4,2 \%-10,1 \%$ \\
\hline Fumantes habituais & 47 & $(15,4 \%)$ & $11,6 \%-20,1 \%$ \\
\hline \multicolumn{4}{|l|}{$\mathrm{IMC}$} \\
\hline Normal & 255 & $(83,6 \%)$ & $78,9 \%-87,5 \%$ \\
\hline Sobrepeso & 44 & $(14,4 \%)$ & $10,8 \%-19,0 \%$ \\
\hline Obeso & 6 & $(2,0 \%)$ & $0,8 \%-4,4 \%$ \\
\hline Total & 305 & $(100 \%)$ & - \\
\hline
\end{tabular}

\begin{tabular}{|c|c|c|c|}
\hline $\begin{array}{c}\text { Contracentivos } \\
\text { orais }\end{array}$ & $N^{\circ}$ & (\%) & $10-95 \%$ \\
\hline Não usuárias & 147 & $(51.4 \%)$ & $45.4 \%-57.3 \%$ \\
\hline Usuárias & 139 & $(48.6 \%)$ & $42.7 \%-54.6 \%$ \\
\hline Total & 286 & $(100 \%)$ & - \\
\hline
\end{tabular}

No Quadro II, estão apresentados os efeitos ajustados do IMC, da Duração do Hábito de Fumar e do Número de Cigarros consumidos/dia,em diferentes níveis de exposição aos antecedentes familiares de doenças crônicas não transmissíveis. Observa-se que, entre os indivíduos com dois ou mais antecedentes familiares, as três variáveis mantiveram associação com os níveis de pressão sistólica $(\mathrm{p}<0,05)$. Para a pressão diastólica, a Duração do Hábito de Fu-

\begin{tabular}{|c|c|c|c|c|}
\hline & & Variá & Nente & \\
\hline Variável & Pressẫo & :a Média & Pressẫo $A$ & lica Média \\
\hline Independente & (ß) & & (B) & \\
\hline & & mbas as & & \\
\hline IDADE & 0,2769 & 0,0359 & 0,2610 & 0,0101 \\
\hline SMOKE & 1,1536 & 0,0346 & 0,4151 & 0,3205 \\
\hline $1 \mathrm{MC}$ & 1,0216 & 0,0000 & 0,8615 & 0,0000 \\
\hline & & ara mulin & & \\
\hline IDADE & 0,0597 & 0,6860 & 0,1052 & 0,3666 \\
\hline SMOKE & 1,1582 & 0,0336 & 0,5970 & 0,1640 \\
\hline$I M C$ & 1,0205 & 0,0000 & 0,8674 & 0,0000 \\
\hline & & ara mulh & & \\
\hline IDADE & 0,0353 & 0,8116 & 0,0964 & 0,4091 \\
\hline $\mathrm{CO}$ & $-1,3023$ & 0,2197 & 0,0954 & 0,9098 \\
\hline IMC & 1,0226 & 0,0000 & 0,8695 & 0,0000 \\
\hline & & ara muih & & \\
\hline IDADE & 0,4137 & 0,7831 & 0,1148 & 0,3302 \\
\hline COTEMP & 0,0055 & 0,9888 & $-0,3207$ & 0,2962 \\
\hline $1 \mathrm{MC}$ & 1,0241 & 0,0000 & 0,8829 & 0,0000 \\
\hline $\begin{array}{l}\text { SMOKE = Hábito de } \\
\text { IMC= Îndoe de ma } \\
\text { CO= Uso de contra } \\
\text { COTEMP = Duracắ }\end{array}$ & rais & suárias & & \\
\hline
\end{tabular}




\section{Ouadro II- Valores Beta ( $\mathcal{A}$ e respectivos valores $(\boldsymbol{D})$ descritivos para as análises de Rearessão} Linear Múltinla. estratificando-se para antecedentes familiares

\begin{tabular}{|c|c|c|c|c|}
\hline \multirow{3}{*}{$\begin{array}{c}\text { Variável } \\
\text { Independente }\end{array}$} & \multicolumn{4}{|c|}{ Variável Dependente } \\
\hline & \multirow{2}{*}{\multicolumn{2}{|c|}{$\begin{array}{l}\text { Pressão Arterial Sistólic a Média } \\
\begin{array}{l}(\beta) \\
\text { (D) }\end{array}\end{array}$}} & \multicolumn{2}{|c|}{ Pressẫo Arterial Diastólica Média } \\
\hline & & & (ß) & (b) \\
\hline \multicolumn{5}{|c|}{ Para ambos os sexos } \\
\hline $1 \mathrm{M} \mathrm{C}_{(\mathrm{B})}$ & 1,5121 & 0,0191 & 0,8281 & 0,1166 \\
\hline $1 M C_{(2)}$ & 0,0230 & 0,9786 & 0,5070 & 0,2031 \\
\hline $\mathrm{IMC}_{(\mathrm{s})}$ & 1,2669 & 0,0346 & 1,3828 & 0,0022 \\
\hline $1 \mathrm{MC}_{(4)}$ & 0,7367 & 0,5964 & 0,5801 & 0,6058 \\
\hline $\mathrm{IMC} \mathrm{C}_{\circledast}$ & 0,5339 & 0,2409 & 0,8646 & 0,2207 \\
\hline $1 M C_{(\bullet)}$ & 1,0511 & 0,0000 & 0,8184 & 0,0000 \\
\hline \multicolumn{5}{|c|}{ Para ambos os sexos } \\
\hline $\mathrm{TF}_{(1)}$ & $-0,7091$ & 0,6408 & $-1,5374$ & 0,2252 \\
\hline$\left.T F_{(}\right)$ & 0,1011 & 0,9385 & $-0,9838$ & 0,0829 \\
\hline$T F$ (§) & $-0,5248$ & 0,4602 & $-0,9211$ & 0,0607 \\
\hline $\mathrm{TF}(\mathrm{a})$ & 2,1975 & 0,0073 & 0,7009 & 0,3982 \\
\hline $\mathrm{TF}_{\text {() }}$ & 0,8855 & 0,0554 & 0,9132 & 0,2339 \\
\hline$T F$ () & 0,7723 & 0,0228 & 0,0307 & 0,9080 \\
\hline$N C_{(1)}$ & $-0,3255$ & 0,6219 & $-0,6239$ & 0,2584 \\
\hline $\mathrm{NC}(2)$ & $-0,0946$ & 0,8673 & $-0,6403$ & 0,0050 \\
\hline $\mathrm{NC}_{(3)}$ & 0,0188 & 0,9838 & $-0,0964$ & 0,8837 \\
\hline $\mathrm{NC}_{(\rightarrow)}$ & 2,9178 & 0,0029 & 1,1813 & 0,2466 \\
\hline$N C_{(9)}$ & 0,4279 & 0,0645 & 0,4794 & 0,2058 \\
\hline$N C_{(6)}$ & 0,4556 & 0,0025 & 0,0517 & 0,6626 \\
\hline \multicolumn{5}{|c|}{ 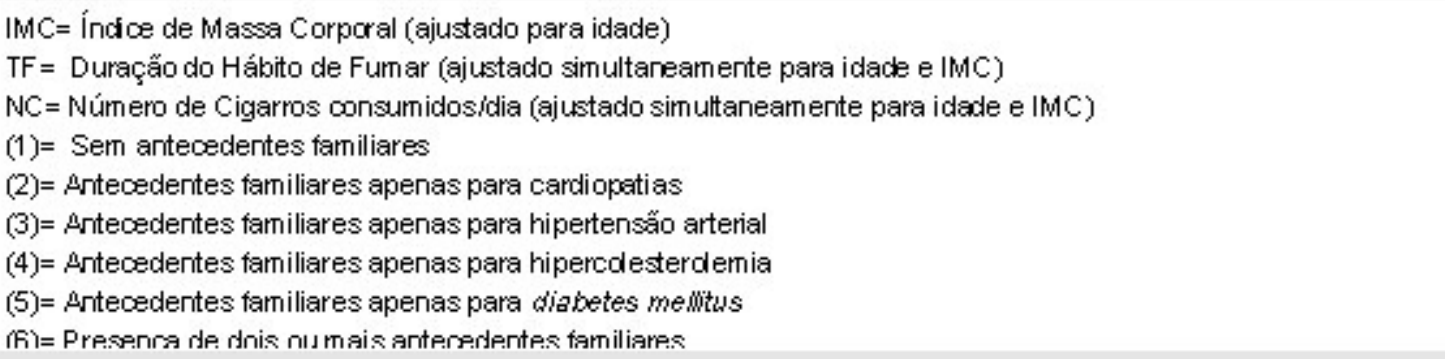 } \\
\hline
\end{tabular}


mar não apresentou significância estatística em nenhuma das categorias de exposição de antecedentes familiares. O IMC, em indivíduos com dois ou mais antecedentes familiares ou entre aqueles com apenas antecedentes familiares de hipertensão arterial, manteve-se associado com os níveis de pressão diastólica. O Número de Cigarros Consumidos/dia apenas esteve associado aos níveis de pressão diastólica entre os indivíduos que referiram antecedentes familiares apenas para cardiopatias.

\section{4- DISCUSSÃO}

No presente estudo, pôde-se constatar que a maioria dos indivíduos estudados eram do sexo feminino, fato este decorrente da história de formação da profissão que esteve, desde sua origem, fundamentada no "cuidado" que, tradicionalmente, tem sido uma atividade predominantemente praticada por mulheres ${ }^{(19)}$.

Com relação aos antecedentes familiares para doenças crônicas (Tabela I), destaca-se uma elevada prevalência de estudantes com dois ou mais antecedentes. Entre aqueles com apenas um antecedente, destacaram-se os estudantes com história familiar de hipertensão arterial. Considera-se oportuno destacar ser mais relevante a busca de antecedentes familiares para enfermidades como o diabetes e a hipercolesterolemia do que a aferição bioquímica desses indicadores, porquanto, em virtude de se tratar de uma população jovem, os indicadores bioquímicos poderiam ainda não ser bons preditores das respectivas enfermidades. É fato reconhecido, entretanto, que a presença de antecedentes familiares para diabetes, hipercolesterolemia, hipertensão arterial, e para doenças cardiovasculares tem relevância para o subseqüente desenvolvimento de tais enfermidades, conforme reportado por vários autores $^{(4,20 / 23)}$. Lauer, $1991^{(23)}$, comentando os resultados do Cardia Study, destacou que 50\% dos descendentes, entre três e trinta anos, de indivíduos com doença isquêmica do coração, eram portadores de alguma forma de dislipidemia, salientando a importância de se considerar a história familiar como indicador precoce de enfermidades crônicas.

Os resultados do presente estudo apontaram uma prevalência de $65,6 \%$ de indivíduos com dois ou mais antecedentes familiares para doenças crônicas, o que coloca tal população como de elevado risco para o desenvolvimento futuro das enfermidades citadas.

Embora a hipertensão arterial em jovens seja um importante fator de risco para as coronariopati- as ${ }^{(6,20)}$, os níveis de variação para a pressão sistólica e diastólica, na população estudada, foram considerados normais (Tabelas II e III). Entretanto, cabe destacar que Ravogli et al., $1990^{(24)}$,estudando universitários com níveis pressóricos $\leq 140$ × $90 \mathrm{mmHg}$, conseguiram estabelecer que, independentemente de variáveis relacionadas ao stress, os níveis mais elevados de pressão arterial, nos estudantes, foram identificados entre aqueles em que a hipertensão arterial foi detectada em ambos os pais, quando comparados aos que tinham apenas um ou nenhum dos pais hipertensos.

Com relação ao hábito de fumar (Tabela IV), destaca-se uma prevalência de fumantes habituais para o conjunto dos indivíduos de 15,4\%. Segundo PratMarin et al.,1994(11), que também obtiveram resultados semelhantes, os conhecimentos sobre os malefícios do fumo, adquiridos durante o curso, parecem não ter exercido influência sobre o hábito.

Considerando, conjuntamente, a prevalência do hábito entre os fumantes habituais e os ocasionais, observou-se para o conjunto uma prevalência de $22 \%$. Rodrigues et al., 1991 ${ }^{(12)}$, estudando a prevalência do hábito de fumar em universitários do Campus de Ribeirão Preto em quatro anos-calendário (1980, 81, 86 e 88), observaram que os estudantes de enfermagem, em 1988, apresentavam as mais elevadas prevalências do hábito, que foram respectivamente $22,2 \%$ para os homens e $20,2 \%$ para as mulheres, quando comparadas às de outras unidades de ensino relacionadas à saúde. Destaca-se, entretanto, que os autores consideraram os fumantes ocasionais como não fumantes, incluindo, na categoria de fumantes, apenas os fumantes habituais.

No presente trabalho, se forem considerados, como fumantes, apenas os fumantes habituais, os dados são sugestivos de um declínio do hábito, entre os estudantes de enfermagem, quando comparados aos achados de Rodrigues et al. ${ }^{(12)}$. Embora a frequiência do hábito ainda permaneça elevada, o suposto declínio pode ser resultante de medidas coercitivas de intimidação aos tabagistas, que vêm se intensificando ao longo dos últimos anos.

Com relação ao índice de massa corporal (Tabela IV) observa-se, para o conjunto dos indivíduos, maior proporção daqueles com IMC dentro dos limites normais $(83,6 \%)$. A prevalência de sobrepeso somada à de obesidade, no estudo (16,4\%), muito se assemelha àquela encontrada por Lolio \& Latorre, $1991^{(25)} \mathrm{em}$ estudo populacional, em Araraquara, na faixa etária entre 18 e 24 anos $(16,1 \%)$. Embora a obesidade este- 
ja condicionada a fatores de ordem cultural e de meio ambiente (sociedades urbanas), os resultados encontrados no estudo diferem daqueles relatados por Rasheed et al ${ }^{(26)}$ que, estudando o IMC em acadêmicos de Enfermagem e Medicina na Arábia Saudita, encontraram uma proporção de 30,6 \% de obesos, denotando que influências culturais e de ordem genética sobre este indicador sejam, talvez, mais relevantes que a concentração em áreas urbanas.

A elevada prevalência de usuárias de contraceptivos orais $(48,6 \%)$, na população estudada, (Tabela $\mathrm{V}$ ) impõe a necessidade de orientação quanto às possíveis conseqüências de seu uso indiscriminado e relacionadas ao aparecimento, no curto ou médio prazo, das doenças crônicas não transmissíveis, conforme o apontado em alguns estudo ${ }^{(7,8,9,27)}$.

Além da detecção da prevalência dos conhecidos fatores de risco para enfermidades crônicas, houve interesse em se investigar, no estudo, possíveis associações de alguns desses fatores com os níveis médios de pressão arterial sistólica e diastólica, após o ajustamento para potenciais fatores de confusão. Os resultados, apresentados no Quadro I, permitiram identificar que o índice de massa corporal constituiu-se em significante preditor dos níveis de pressão arterial sistólica e diastólica, tanto no conjunto da população (ambos os sexos) quanto na população feminina $(\mathrm{p}<0,05)$. O hábito de fumar esteve associado, apenas, com os níveis médios da pressão sistólica. Fonseca et al., 1995(6), estudando a influência de alguns fatores de risco sobre a pressão arterial em acadêmicos da Universidade de Taubaté, não detectaram diferenças estatisticamente significantes entre as respectivas médias da pressão arterial sistólica ou diastólica em indivíduos expostos e não expostos ao tabagismo. Embora o autor tenha baseado os resultados em análises univariadas, foi possível detectar interação entre a obesidade e o tabagismo sobre os níveis de pressão arterial diastólica.

$\mathrm{Na}$ população feminina do presente estudo, o uso de contraceptivos orais ou sua duração não apresentaram associação com os níveis médios de pressão sistólica ou diastólica ( $p>0,05)$. A comparação com outros achados da literatura fica, entretanto, prejudicada pela escassez de estudos com propostas semelhantes.

Num segundo momento, foram efetuadas análises, objetivando-se investigar a associação entre os principais preditores (IMC e Hábito de Fumar) e os níveis de pressão arterial sistólica e diastólica, nas di- ferentes categorias de exposição aos antecedentes familiares (Quadro II). Nessa análise, os fumantes foram classificados segundo o tempo de exposição ao hábito e o número de cigarros consumidos/dia. Os resultados revelaram que o índice de massa corporal, o tempo de fumo e o número de cigarros consumidos/ dia foram significantes preditores dos níveis de pressão arterial sistólica $(\mathrm{p}<0,05)$ na presença de dois ou mais antecedentes familiares. Para a pressão arterial diastólica e, entre os indivíduos com dois ou mais antecedentes familiares, apenas o índice de massa corporal, foi considerado um significante preditor $(\mathrm{p}<0,05)$. Burke et al. ${ }^{(20)}$, analisando dados do Cardia Study em indivíduos entre 18 e 30 anos de idade, investigaram a existência de associações entre os níveis de alguns fatores de risco para doenças crônicas e a presença de um ou mais antecedentes familiares para essas condições. Os resultados evidenciaram que os níveis de pressão arterial sistólica, pressão diastólica e o índice de massa corporal estiveram associados com a exposição a dois ou mais antecedentes familiares para doenças crônicas e com a exposição a antecedentes familiares apenas para hipertensão arterial. Esses resultados se assemelham aos do presente estudo, e ambos destacam a relevância dos antecedentes familiares como indicadores precoces da presença ou da associação de outros fatores de risco para as doenças crônicas não transmissíveis.

No que pesem as limitações impostas quanto ao controle de fatores de risco que dependem de herança genética, torna-se fundamental investigar, o mais precocemente possível, a presença de antecedentes familiares para as doenças crônicas não transmissíveis, por sua atuação como indicadores de outros fatores de risco para tais enfermidades.

Por outro lado, faz-se também necessária a organização de estratégias efetivas de controle do tabagismo, da obesidade e do uso de contraceptivos orais na falange universitária analisada, levando-se em consideração a longa latência que caracteriza as doenças crônicas ao lado do envelhecimento populacional progressivo, decorrente do atual estágio da transição demografica e epidemiológica ${ }^{(28,29)}$.

\section{AGRADECIMENTOS}

Aos enfermeiros, Andreia Perseguin de Souza, Gabriela Eneida Françolin Silva, Luzia Elaine Galdeano e Rodrigo Trassi de Araújo, pela colaboração junto à coleta de dados do presente trabalho. 
MORAES SA; MEIRA L \& FREITAS ICM. Risk factors for non-communicable diseases in nursing students from Ribeirão Preto-Brazil. Medicina; Ribeirão Preto, 33: 312-321, july/sept. 2000

ABSTRACT: Purpose: The purpose of this investigation was to study the risk factors prevalence to chronic diseases among students from the University of São Paulo at Ribeirão Preto College of Nursing, Brazil, and evaluate the association among those risk factors and systolic and diastolic blood pressure.

Methods: The population was formed by 305 nursing students registered in 1995, the data collection was based on an standardized interview carried out between August and December 1995. Besides social-demographic conditions, familial history for non-communicable diseases, blood pressure, smoking, obesity and oral contraceptive consumption were also investigated. The prevalence of risk factors was estimated with $95 \%$ confidence limits. The association among them was evaluated by multiple linear regression at a 0.05 significance level.

Results: The prevalence of two or more familial antecedents for chronic diseases was $65.6 \%$ (Cl: $59.9 \% 3 / 470.8 \%)$. The smoking prevalence was $15.4 \%(\mathrm{Cl}: 11.6 \% 3 / 420.1 \%)$ and oral contraceptive consumption prevalence was 48.6\% ( $\mathrm{Cl}: 42.7 \% 3 / 454.6 \%)$. The Body Mass Index (BMI) adjusted by age and smoking was an important predictor to systolic and diastolic pressure levels $(p<0.05)$. Among females BMI adjusted by age and contraceptives use or duration of contraceptives use showed association with systolic and diastolic pressure levels $(p<0.05)$. Among individuals with two or more familial antecedents for chronic diseases, BMI, duration of smoking and daily number of cigarettes consumed showed association with systolic pressure levels $(p<0.05)$. BMI was associated with diastolic blood pressure among individuals with two or more familial antecedents or those with hypertension antecedents only $(p<0.05)$. The daily number of cigarettes consumed showed association with diastolic blood pressure among individuals with familial antecedents for cardiovascular diseases $(p<0.05)$.

Discussion: Authors make considerations about the influence of environment and genetic risk factors for chronic diseases and recommend some strategies in order to control smoking, obesity and oral contraceptive use in this population stratum.

UNITERMS: Prevalence. Risk Factors. Chronic Disease

\section{REFERÊNCIAS BIBLIOGRÁFICAS}

1 - REGO RA; BERARDO FA; RODRIGUES SS; OLIVEIRA ZM; OLIVEIRA MB; VASCONCELOS C; AVENTURATO LV; MONCAU JE \& RAMOS LR. Fatores de risco para doenças crônicas não transmissíveis: inquérito domiciliar no município de São Paulo, Brasil: metodologia e resultados preliminares. Rev Saúde Pública 24: 277-285,1990.

2 - INCLEN MULTICENTRE COLLABORATIVE GROUP. Risk factors for cardiovascular disease in the developing world. A Multicentre Collaborative Study in the International Clinical Epidemiology Network (INCLEN). J Clin Epidemiol 45: 841-847, 1992.

3 - MALERBI DA \& FRANCO LJ. Multicenter study of the prevalence of diabetes mellitus and impaired glucose tolerance in the urban Brasilian population aged 30-69 years. The Brazilian Cooperative Group on the Study of Diabetes Prevalence. Diabetes Care 15: 1509-1516, 1992.

4 - TOMONO S; OHSHIMA S \& MURATA K. The risk factors for ischaemic heart disease in young adults. Jpn Circ J 54:436441, 1990.

5 - TANAJURA LF; PIEGAS LS; TIMERMAN A; RAMOS RF; GUN C; TIMERMAN S; ABDALLA LA; FREIRE BB; ROMANO ER \& SOUSA JE. Infarto agudo do miocárdio em pacientes com idade inferior a 40 anos. Arq Bras Cardiol 55:237-240, 1990.
6 - FONSECA LR; DA SILVA FT; NATIVIDADE JE \& SCHIMIDT LO. Estudo da prevalência da hipertensão arterial em acadêmicos do Departamento de Ciências Médicas da Universidade de Taubaté. Arq Bras Cardiol 64:553-555, 1995

7- BERAL V; HERMON C; KAY C; HANNAFORD P; DARBY S \& REEVES G. Mortality associated with oral contraceptive use: 25 year follow up of cohort of 46.000 women from Royal College of General Practioners' oral contraception study. BMJ 318: 96-100, 1999

8 - TIMMER A; SUTHERLAND LR \& MARTIN F. Oral contraceptive use and smoking are risk factors for relapse in Crohn's disease. The Canadian Mesalamine for Remission of Crohn's Disease Study Group. Gastroenterology 114: 1143-1150, 1998.

9 - SANCHEZ-GUERRERO J; KARLSON EW; LIANG MH; HUNTER DJ; SPEIZER FE \& COLDITZ GA. Past use of oral contraceptives and the risk of developing systemic lupus erythematosus. Arthritis Rheum 40: 804-848, 1997.

10 - MORAES SA \& SOUZA JMP. Diabetes Mellitus e doença isquêmica do coração: estudo tipo caso-controle. Rev Saúde Pública 30: 364-371,1996.

11 - PRAT-MARIN A; FUENTES-ALMENDRAS MM; SANZGALLEN P; CANELA-ARGUES R; CANELA-SOLER J; PARDELL-ALENTA H \& SALLENAS-SANMARTI LL. Epidemiologia del tabaquismo in los estudiantes de ciencias de la 
salud. Rev Saúde Pública 28: 100-106, 1994.

12 - RODRIGUES SEM \& RUFFINO-NETTO A. Tendência do tabagismo entre estudantes da Universidade de São Paulo, Campus de Ribeirão Preto, no período de 1980-1988. Medicina, Ribeirão Preto 24:149 -158, 1991.

13 - HENNEKENS CH \& BURING JE. Epidemiology in medicine. Little Brown, Boston, 1987.

14 - BRAY GA. Obesity: basic considerations and clinical approaches. Dis Mon Chicago, 35(7) 1989.

15 - FOX HOLDING INC. Foxpro, vs. 2.0. Pat. Pend. American Edition, New York 1991.

16 - DEAN J; DEAN A \& FRANCO E. Epiinfo-computer programs for Epidemiology. Division of Surveillance and Epidemiologic studies, Epidemiology Programs Office, Center for Disease Control, Atlanta 1990.

17 - KLEINBAUM DG; KUPPER LL \& MULLER KE. Applied regression analysis and other multivariable methods. PWSKENT Publishing, Boston 1988.

18 - SPSS INC. Statistical Package in Social Science: Release 7.0. Copyright $^{\odot}$ London 1996.

19 - PAIXÃO W. História da enfermagem. 5a. ed. Julio C. Reis, Rio de Janeiro 1979.

20 - BURKE GL. Relation of risk factor levels in young adulthood to parentl history of disease. The CARDIA Study. Circulation 84: 1176-1187, 1991.

21 - ARNOLD AZ \& MOODIE DS. Coronary artery disease in young women: risk factor analysis and long term follow-up. Cleve. Clin J Med 60: 393-398, 1993.
22 - COLDITZ GA. A prospective study of parental history of miocardial infartion and coronary heart disease in women. Am J Epidemiol 123:48-58, 1986.

23 - LAUER RM. A family history of risk factors and cardiovascular diseases. Circulation 84: 1445-1446, 1991.

24 - RAVOGLI A; TRAZZI S; VILANI A; MUTTI E; CUSPIDIC C; SAMPIERI L; DE AMBROGGI L; PARATI G; ZANCHETTI A \& MANCIA G. Early 24-hour blood pressure elevation in normotensive subjects with parental hypertension. Hipertension 16: 491-497, 1990.

25 - LOLIO CA \& LATORRE MRDO. Prevalência de obesidade em localidade do estado de São Paulo, Brasil, 1987. Rev Saúde Pública 25: 33-36, 1991.

26 - RASHEED P; ABOU-HOZAIKA BM \& KHAN A. Obesity among young Saudi Female Adults: a prevalence study on medical and nursing students. Public Health 108: 289-294, 1994.

27 - WHO Collaborative Study of Cardiovascular Disease and Steroid Hormone Contraception. Acute myocardial infarction and combined oral contraceptives: results of na international multicentre case-control study. Lancet 349: 1202-1209, 1997.

28 - THE EPIDEMIOLOGICAL TRANSITION.[Editorial] Lancet 2: 670,1977

29 - OMRAM AR. The epidemiologic transition in the Americas. PAHO-The University of Maryland at College Park, Washington, 1996.

Recebido para publicação em 20/06/1999

Aprovado para publicação em 11/07/2000 